\title{
An ERP investigation of location invariance in masked repetition priming
}

\author{
Stéphane Dufau and Jonathan Grainger \\ CNRS and Université de Provence, Marseille, France \\ AND \\ Phillip J. Holcomb \\ Tufts University, Medford, Massachusetts
}

\begin{abstract}
In an experiment combining masked repetition priming and the recording of event-related potentials (ERPs) the location of prime stimuli relative to centrally located target words was manipulated. Prime words could appear at the same location as targets or shifted one letter position to the right or to the left. Repetition priming effects (amplitude differences across the repeat vs. unrelated prime conditions) were found in a series of ERP components starting at around $100 \mathrm{msec}$ posttarget onset. The earliest of these, the N/P150 component, was found to be sensitive to prime location. Repetition priming was only apparent with centrally located primes in this component. Repetition priming effects in later components (N250 and N400), on the other hand, were not affected by prime location. The results are interpreted in terms of location-specific letter detectors that map onto a higher level, location-invariant orthographic code for printed words.
\end{abstract}

Over the last two decades, masked priming has become a central tool for behavioral research aimed at decomposing the fast, highly automatized processing performed by skilled readers during visual word comprehension. In recent work, this technique has been combined with the recording of event-related potentials (ERPs) in order to provide a detailed analysis of the time course of component processes, and to provide some insight into the neural mechanisms involved. This research, combining ERP recordings and masked repetition priming of visual word stimuli, has outlined a cascade of ERP components sensitive to the priming manipulation (Holcomb \& Grainger, 2006; Kiyonaga, Grainger, Midgley, \& Holcomb). As well as the classic N400 component, Holcomb and Grainger described two earlier components that are of particular relevance for the present study. We will first summarize these findings.

The first of these, the P150 (here referred to as the $\mathrm{N} / \mathrm{P} 150$ ), started as early as $90 \mathrm{msec}$ and was over by 180 msec. This was a spatially focal component that produced priming effects that were positive-going at occipital sites (especially over the right hemisphere) and negative-going at more anterior sites (priming here refers to targets that are unrelated to the prior prime, compared to targets that were repetitions of the prime). The distributional and temporal similarity of this effect across several studies using words (Holcomb \& Grainger, 2006), single letters (Petit, Midgley, Holcomb, \& Grainger, 2006) and pictures of objects (Eddy, Schmid, \& Holcomb, 2006) suggests that it reflects an early process, possibly one involved in mapping visual features onto higher level form representations (Grainger \& Holcomb, in press). The slightly later N250 described by Holcomb and Grainger (2006) started around $180 \mathrm{msec}$ and ended close to $300 \mathrm{msec}$, and produced a more widely distributed effect that was somewhat larger over more anterior scalp sites than over the most posterior sites. This component took the form of a larger negativity to target words that were unrelated to their preceding prime words (e.g., phone-TRUCK), intermediate for targets that overlapped with primes in all but one letter position (e.g., trock-TRUCK), and smallest for complete repetitions (e.g., truck-TRUCK). Grainger and Holcomb (in press) have argued that such N250 effects, which were not seen for individual letters (Petit et al., 2006) or objects (Eddy et al., 2006), reflect the process whereby prelexical orthographic representations (letters and letter clusters) are mapped onto whole-word orthographic representations.

These two early ERP components are particularly relevant for the present study, given the focus on the earliest phases of processing in visual word recognition - those involved in mapping location-specific feature information onto abstract letter identities, and from these onto a position-invariant orthographic code. These processes were described by Grainger and van Heuven (2003) in their model of orthographic processing couched within the framework of a generic interactive-activation model (McClelland \& Rumelhart, 1981). In this model, shown in Figure 1, a bank of location-specific letter detectors re- 


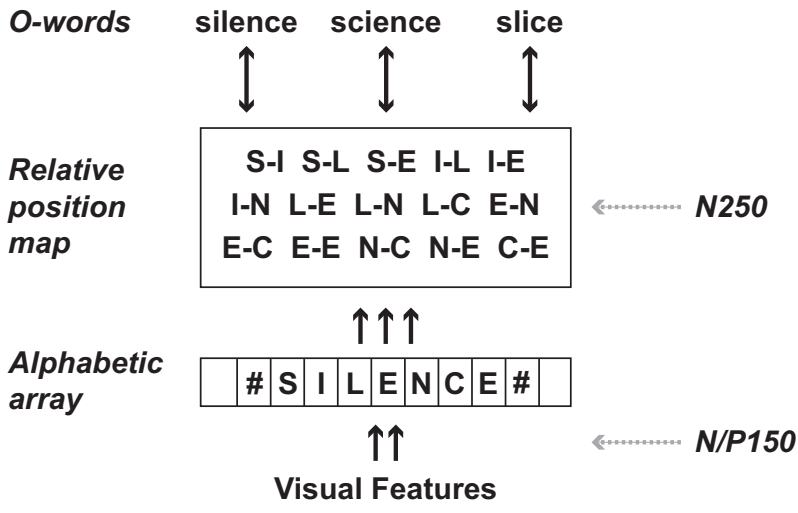

Figure 1. Grainger and van Heuven's (2003) model of orthographic processing. A letter string is first processed by a bank of alphabetic character detectors (the alphabetic array). The next level of processing combines information from different processing slots in the alphabetic array to provide a relative position code for letter identities. These relative-position coded letter identities control activation at the level of whole-word orthographic representations ( $\mathrm{O}$-words) via bidirectional excitatory connections with all units at the relative position level. The figure also shows a tentative proposal for mapping two ERP components (N/P150 and N250) onto this functional architecture.

ceive featural information extracted from the stimulus and send activation onto location-invariant representations that code for the relative position of letters within the stimulus (see Caramazza \& Hillis, 1990, for a similar proposal). Grainger and van Heuven proposed "open-bigrams," contiguous and noncontiguous ordered letter combinations, as a mechanism for computing a location-invariant relative position orthographic code (see also Whitney, 2001). It is this prelexical orthographic code that then activates whole-word orthographic representations, which in turn activate semantic representations. This general approach was recently adopted and extended by Dehaene, Cohen, Sigman, and Vinckier (2005) in their model of visual word recognition. Dehaene et al. (2005) described a more graded hierarchical approach that was only implicit in the simplified picture offered by Grainger and van Heuven (2003; see also Grainger, Granier, Farioli, Van Assche, \& van Heuven, 2006).

One key element in Grainger and van Heuven's model is the alphabetic array, a hypothesized bank of letter detectors that perform parallel, independent letter identification. These letter detectors are assumed to be invariant to the physical characteristics of letters (both their size and their shape), but not invariant to position. According to this model, featural information at a given location along the horizontal meridian is mapped onto abstract letter representations that code for the presence of a given letter identity at that particular location. These abstract letter representations are thought to be activated equally well by the same letter written in different case, in a different font, or in a different size (within a given level of tolerance for variations in size). The next stage of processing, referred to as the relative-position map, is thought to code for the relative (within-stimulus) position of letter identities independently of their shapes and sizes, and independently of the location of the stimulus word (location invariance).

As previously mentioned, Grainger and Holcomb (in press) tentatively associated the N/P150 ERP component with the mapping of visual features (letter parts) onto letter representations in the alphabetic array, and the N250 ERP component with processing at the level of positioninvariant prelexical orthographic representations. Therefore, according to this account, the N/P150 component should be sensitive to shifts in location across prime and target stimuli, whereas the N250 component should not. The present experiment tests these predictions by manipulating the position of prime stimuli relative to centrally located target stimuli. Primes could be at the same location as targets or shifted one letter space to the right or to the left, with primes being the same word as targets in the repetition prime condition, and different words in the unrelated prime condition. We expect the manipulation of the prime location to modulate the effects of repetition priming in the N/P150 ERP component. On the other hand, priming effects in the N250 ERP component, and a fortiori any later components, should not be affected by prime location.

\section{METHOD}

\section{Participants}

Thirty-three healthy volunteers (average age, 22.8 years; 12 females) from the University of Provence took part in the experiment as paid volunteers. All participants were right-handed native speakers of French and reported having normal or corrected-to-normal vision.

\section{Design and Stimuli}

Four hundred eighty words were selected from the French lexical database Lexique (New, Pallier, Brysbaert, \& Ferrand, 2004). The words were four to six letters in length (average length, 5.28 letters), and their printed frequency ranged between 10 and 50 occurrences per million. Thirty of these words were animal names used as probe items on noncritical trials. The remaining 450 words served as primes and targets on critical trials. A $2 \times 3$ within-participants factorial design was constructed, with repetition (prime is the same word as target, or a different word) and prime location (prime displayed in the center of the screen at the same location as targets, shifted by one letter position to the left or one letter position to the right) as main factors. Stimuli were counterbalanced using nine different lists such that each word was tested as a target in each of the six experimental conditions in six different lists and also served as unrelated primes in three other lists. Within a given list, each word occurred only once, either as target or as prime. Note that this design ensures that grand average ERP comparisons between conditions always involve the same items (primes and targets) in each experimental condition. Stimuli were randomly distributed in three blocks, allowing participants to rest between blocks. Each block consisted of 100 critical trials (50 repetition trials and 50 unrelated trials) and 10 probe trials with animal names serving as probes ( $10 \%$ of the trials).

\section{Procedure}

On each trial, participants were presented a pair of words in a masked priming paradigm (see Figure 2) and were asked to decide whether the target word was an animal name or not by pressing a response button whenever they saw an animal name. Animal names appeared every 10 trials, on average. For all other items, no overt response was required (go/no-go procedure). After completing informed consent and handedness forms, participants were seated comfortably in a sound-attenuated and dimly illuminated room, 


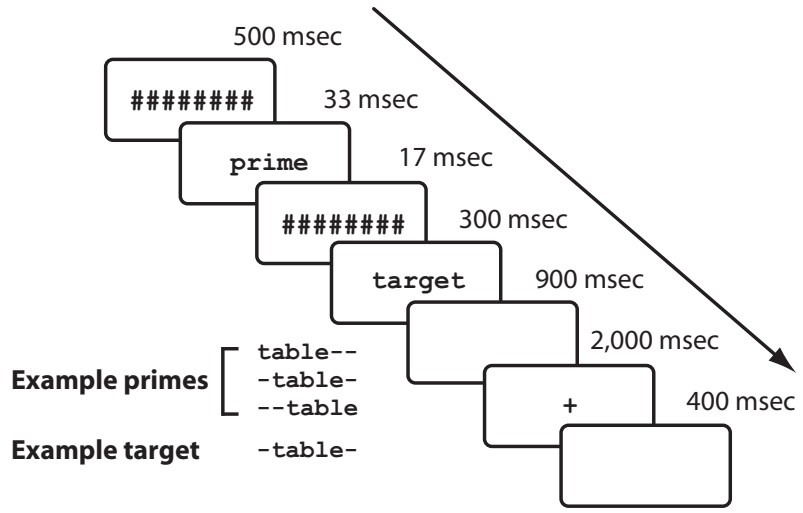

Figure 2. Schematic of the priming procedure used in the present experiment. Location of the prime stimulus relative to the target stimulus is indicated using minus signs, which were not displayed during the experiment.

facing a 17-in. CRT monitor $90 \mathrm{~cm}$ in front of them. Stimuli were displayed as white letters on an $800 \times 600$ pixel black background. All words were presented in lowercase (18-point courier new font). Each trial consisted of a 500-msec forward mask, a 33-msec prime word, a 17-msec patterned backward mask, a 300-msec target word, a 900 -msec blank screen, and a 2,000-msec stimulus indicating that blinking was allowed. The intertrial interval (ITI) was $400 \mathrm{msec}$. The experiment was preceded by a set of practice trials (18 nonanimal and two animal target words), none of which appeared in the actual experiment. The whole testing session lasted $2 \mathrm{~h}$, including short breaks after each block.

\section{Prime Visibility Test}

After the main experiment, participants were given a prime visibility test on which they were instructed to categorize prime stimuli as animal words or not in a forced choice procedure, while ignoring the visible target words. The word stimuli used in the prime visibility test did not appear in the main experiment but had the same length and frequency. Signal detection sensitivity $\left(d^{\prime}\right)$ values were calculated for each participant $(M=-0.026, S D=0.27)$. A test of the distribution of $d^{\prime}$ values against a zero reference showed no significant difference $[t(24)=0.48, p>.1]$, indicating that participants responded at chance level.

\section{ERP Recording}

The EEG activity was recorded continuously through the ActiveTwo Biosemi system from 64 electrodes mounted on an elastic cap (Electro-Cap Inc.) and positioned according to the 10-10 international system (American Clinical Neurophysiology Society, 2006). Two additional electrodes (CMS/DRL nearby PZ) were used as an online reference (for a complete description, see Schutter, Leitner, Kenemans, \& van Honk, 2006; www.biosemi.com). The montage included 10 midline sites and 27 sites over each hemisphere (see Figure 3). Four additional electrodes were used to monitor eye movements and blinks (two placed at lateral canthi and two below the eyes), and two additional electrodes were used for an offline rereferencing (placed behind the ears on mastoid bone). Continuous EEG was digitized at $256 \mathrm{~Hz}$ and filtered offline (20 Hz low-pass, $24 \mathrm{~dB} /$ octave) using EEGLAB software (Delorme \& Makeig, 2004). All scalp sites were rereferenced to the left mastoid offline. The ERP data were time-locked to target presentation and were recorded for 800 -msec posttarget onset with a $100-\mathrm{msec}$ pretarget baseline. Epochs with eye movements, blinks, or electrical activities greater than $\pm 50 \mu \mathrm{V}$ were rejected. Data from participants committing more than $25 \%$ errors in the animal detection task were not considered for analysis. To maintain an acceptable signal-to-noise ratio, a lower limit of 30 artifact-free trials per participant per condition was set.
On this basis, 8 participants were discarded from further analysis, leaving the data of 25 participants for analysis. Overall, $11.9 \%$ of the trials were rejected.

\section{ERP Analysis}

A multifactor repeated measures design was used to analyze ERP data, with mean amplitude as dependant variable. The main analysis included the factor repetition (prime and target were the same word vs. different words) and prime location (prime shifted by one letter to the right vs. prime shifted by one letter to the left vs. prime not shifted). Measurement windows were determined from inspection of the grand average waveforms and included 90 to $180 \mathrm{msec}$ for the N/P150; 200 to $280 \mathrm{msec}$ for the N250; and 350 to $450 \mathrm{msec}$ for the N400. These data were then analyzed using a columnar set of four ANOVAs (Holcomb, Reder, Misra, \& Grainger, 2005; see Figure 3), which allowed a distributional analysis of effects over the head, including hemispheric and anteroposterior factors. This gave a 2 (repetition) $\times 3$ (prime location) $\times 2$ (hemisphere) $\times 2$ (anteroposterior) design. As in Chauncey, Holcomb, and Grainger (2008), an additional regional analysis of the N/P150 component was performed at the FP2/O2 electrode pair. ANOVAs were performed using the Greenhouse-Geisser epsilon correction for nonsphericity, where appropriate (Jennings \& Wood, 1976).

\section{RESULTS}

\section{0-180 msec Posttarget}

There was a significant repetition $\times$ prime location $\times$ anteroposterior interaction at column $4[F(2,48)=3.63$, $p=.03]$. This interaction reflects the fact that repetition priming effects were only robust when primes occupied the same central location as did targets [repetition $\times$ anteroposterior interaction for central primes, $F(1,24)=12.22$, $p=.002]$. In this condition, when unrelated and repeated primes were compared, there was a more negative-going effect in frontal electrodes and a more positive-going effect in occipital electrodes. The repetition $X$ anteroposterior interaction was not significant when primes were shifted to the left or to the right ( $p>.3$; see Figure 4). Based on our previous studies showing the largest N/P150

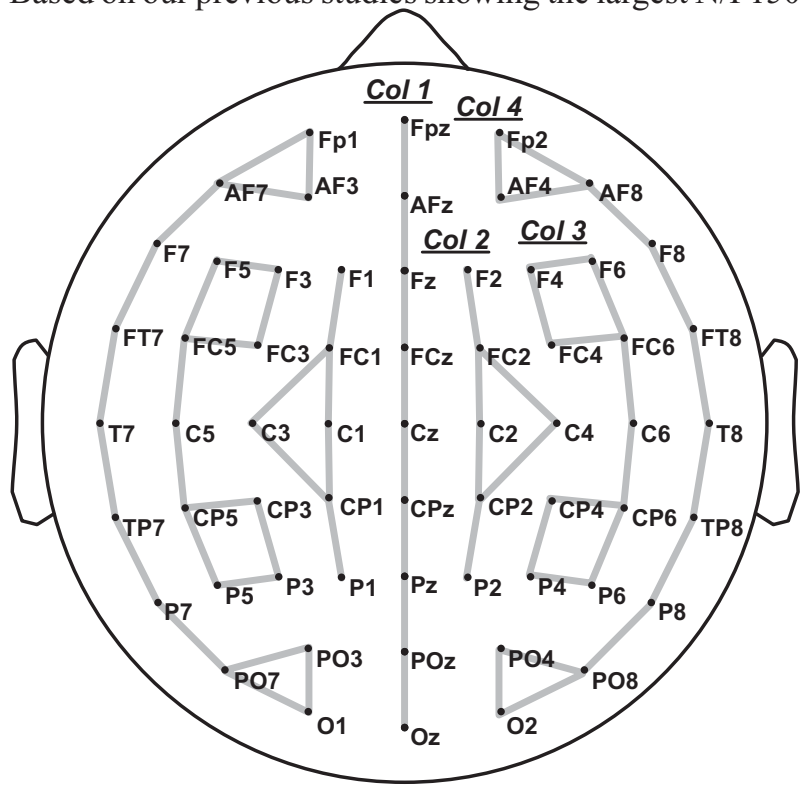

Figure 3. Electrode montage (standard 10-10 system) arranged in four columns (Col. 1 to Col. 4) used in the ERP analysis. 
effects at the right-hemisphere frontal and occipital sites (e.g., Chauncey et al., 2008), we did follow-up analyses restricted to these locations. There was a significant repetition $\times$ location $\times$ anteroposterior interaction at those sites $[F(2,48)=3.78, p=.03]$, whereas the repetition $\times$ location $\times$ hemisphere interaction was not significant $(p=$ .12). The repetition $\times$ location interaction was significant at $\mathrm{O} 2[F(2,48)=3.99, p=.03]$, whereas no interaction was found at FP2. At O1, the repetition $\times$ location interaction was not significant $(p=.44)$. Further follow-up analyses at $\mathrm{O} 2$ revealed that there was a repetition priming effect when primes were not shifted $[F(1,24)=16.10$, $p<.001]$, but no significant priming effect for the two shifted prime conditions $(p>.6)$.

\section{0-280 msec Posttarget}

In this time window, unrelated primes produced a more negative-going waveform than did repetition primes (see Figure 5). A main effect of repetition was found at each column [column $1, F(1,24)=9.60, p=.005$; column 2, $F(1,24)=8.16, p=.008$; column $3, F(1,24)=10.24$, $p=.003$; column $4, F(1,24)=10.97, p=.002]$, and a repetition $\times$ anteroposterior interaction at column 1 and column 2 [column $1, F(1,24)=8.68, p=.007$; column 2 , $F(1,24)=10.25, p=.003]$. These interactions were due to the repetition effect being more prominent in central and more anterior sites. Repetition priming did not interact with prime location in this time window $(p>.8)$.

\section{0-450 msec Posttarget}

Unrelated primes again generated a more negativegoing waveform than did repetition primes in this time window $[F(1,24)=6.74, p=.02]$ (see Figure 5). All columns showed a repetition effect except for column 4 [column $1, F(1,24)=6.61, p=.02$; column $2, F(1,24)=$ $12.22, p=.001$; column $3, F(1,24)=8.40, p=.008]$. A repetition $\times$ anteroposterior interaction was found at column $1[F(1,24)=5.25, p=.03]$ and column $2[F(1,24)=$
$8.30, p=.008]$, due to a greater repetition effect in anterior sites. Again, repetition priming did not interact with prime location in this time window $(p>4)$.

\section{DISCUSSION}

In the present study, we examined effects of prime location on masked repetition priming with word stimuli. Targets were always centrally located, and primes could appear at the same location as targets or shifted one letter to the right or to the left. Repetition priming was found in two early components, peaking at around 150 and $250 \mathrm{msec}$ after target onset, and in one later component, peaking at around $400 \mathrm{msec}$ posttarget onset, corresponding to the N/P150, N250, and N400 components described in previous work (e.g., Chauncey et al., 2008; Holcomb \& Grainger, 2006; Kiyonaga et al., 2007). Most important, we found that the N/P150 component was sensitive to prime location, with repetition effects only being apparent when primes and targets were physically aligned. A very slight horizontal displacement of prime stimuli disrupted repetition effects in this early component. On the other hand, the N250 and the later N400 ERP component showed repetition priming effects not significantly affected by shifts in prime location. This result suggests that the N250 and N400 components reflect processing that occurs at or beyond a location-invariant orthographic code. Furthermore, the pattern of priming effects in the N250 and N400 components is in line with recent behavioral evidence showing that small shifts of prime stimuli $\left( \pm 2^{\circ}\right)$ to the left or right of centrally located targets had a relatively minor impact on repetition priming effects (Marzouki \& Grainger, 2008). However, priming effects were found to diminish with larger prime eccentricities in the Marzouki and Grainger study, suggesting limitations in our capacity to integrate information across spatially distinct objects.

Within the framework of Grainger and van Heuven's (2003) model of orthographic processing, the location-

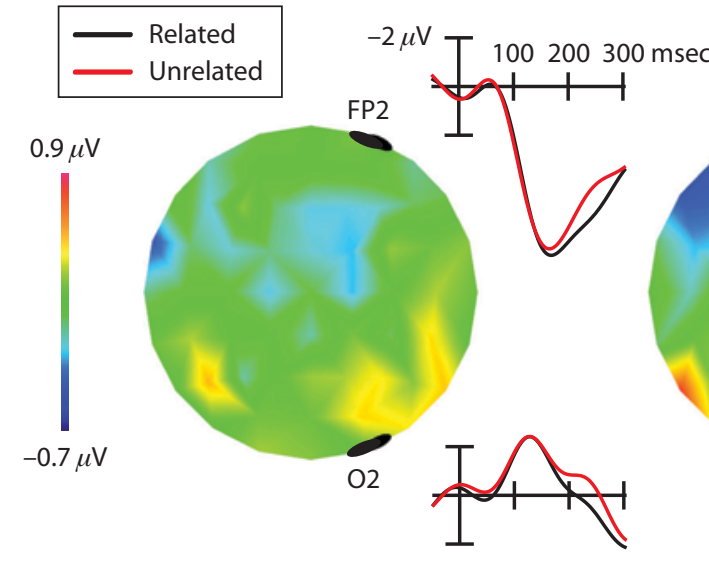

(a)

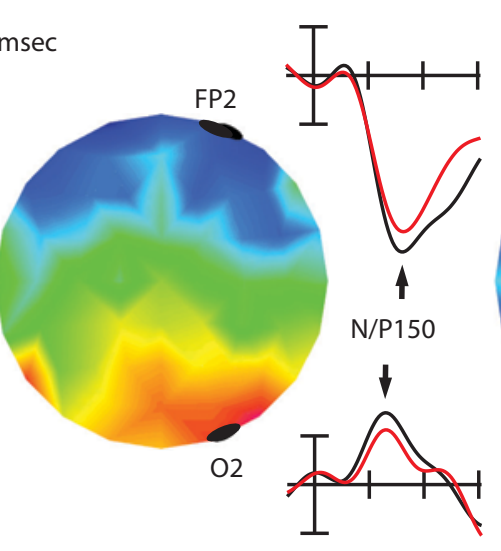

(b)

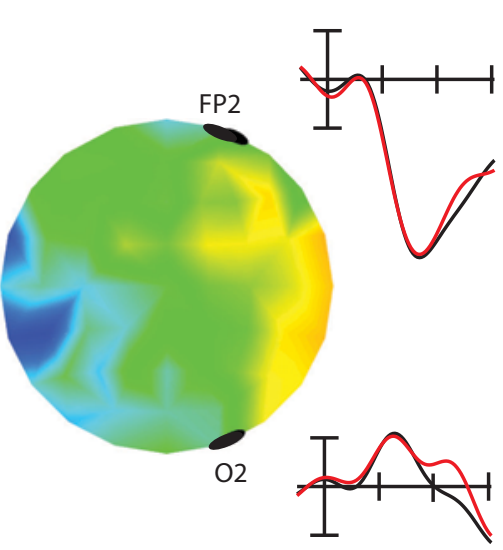

(c)

Figure 4. Effects of repetition priming on the N/P150 ERP component. Scalp maps represent mean amplitude differences per electrode site in the 90- to 180-msec time window between the repetition (black line) and unrelated (red line) conditions for the three different prime locations: Primes are shifted by one letter to the left (a), at the center (b), or shifted by one letter to the right (c). For each condition, ERP waveforms at electrodes FP2 and $\mathrm{O} 2$ are displayed. 
A
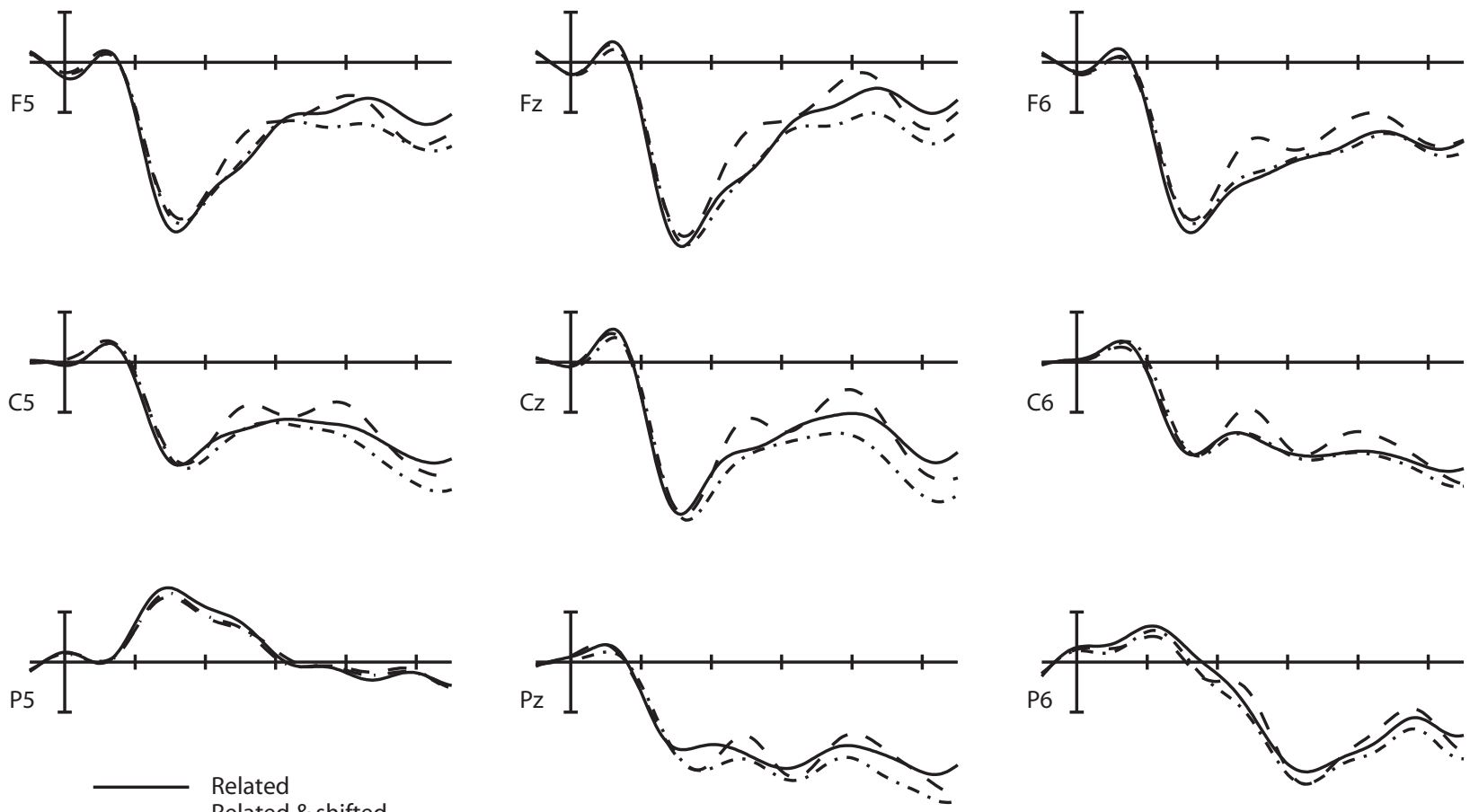

\begin{tabular}{ll}
- & Related \\
\hline$\ldots \ldots$ & Related \& shifted
\end{tabular}

- - - Unrelated

B

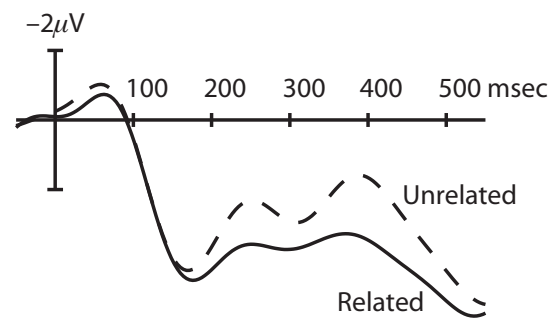

(a)

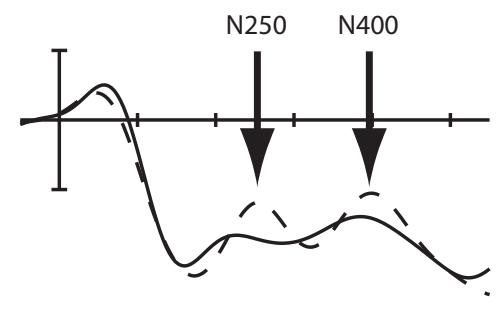

(b)

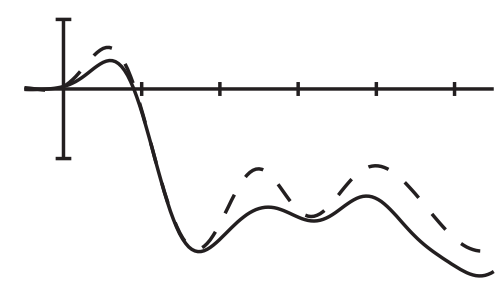

(c)

Figure 5. Panel A: ERP waveforms for nine electrode sites averaged across all unrelated conditions (dashed line), centrally located repetition primes (full line), and repetition primes displaced either to the left or to the right (dashed and dotted line). Panel B: More detailed picture from electrode site CP1 showing repetition priming effects when primes are shifted by one letter to the left (a), displayed at the center (b), or shifted by one letter to the right (c).

specific letter detectors in the alphabetic array would provide the appropriate sensitivity to small horizontal shifts in position found in the N/P150 ERP component in the present study. We therefore tentatively conclude that the N/P150 found in the present study reflects the mapping of visual feature information onto such locationspecific letter representations (see Figure 1). What other information do we have concerning the nature of such location-specific letter representations? In a recent ERP study, Chauncey et al. (2008) found that repetition effects in the N/P150 component were sensitive to whether or not the prime and target were presented in the same or a different font. In contrast, priming effects in later ERP components were not sensitive to the font manipulation across prime and targets. These results suggest that at least part of the process that maps letter features onto location-specific letter representations is not invariant to shape.

We expect the mapping of visual features onto locationspecific letter representations to be a multistage process that involves letter parts that differ as a function of letter case and font, and possibly also involving case-specific letter representations (Petit et al., 2006). The endpoint of this multistage process would be the activation of font and caseinvariant, location-specific letter representations dedicated to the processing of strings of letters. As shown in Figure 1, we hypothesize that the N/P150 component reflects processing along this pathway from visual features to shapeinvariant but location-specific letter representations. Thus, the sensitivity of the N/P150 to visual similarity across 
prime and target (Chauncey et al., 2008) would be a reflection of the earliest phases of this multistage process.

We would also tentatively argue that the evidence we found for location sensitivity in the N/P150 ERP component in the present study likely reflects a set of highly specialized processes specific to orthographic processing. One recent proposal (Tydgat \& Grainger, 2007) is that letter detectors in the alphabetic array have relatively narrow receptive fields (compared to other types of visual symbols that typically do not appear in strings), enabling efficient processing of such stimuli in the highly crowded conditions imposed by letter strings. It is the hypothesized reduced extent of the receptive fields of letter detectors that could explain the high sensitivity of letter strings to horizontal displacement during the earliest phases of orthographic processing. Future research will put this hypothesis to the test by examining effects of prime location on masked repetition priming with isolated letters and other types of visual object.

With respect to the possible anatomical locations of these different processes, an fMRI study by Dehaene et al. (2004) provided evidence that location-invariant orthographic processing is achieved somewhere between posterior and middle left fusiform gyrus (within the region referred to as the visual word-form area; Cohen et al., 2000). Dehaene et al. found a posterior-anterior subdivision within the left fusiform gyrus, depending on whether or not repetition priming was sensitive to relative prime-target location (primes were either at the same location as targets or shifted horizontally by one letter, as in the present study). The most posterior part of fusiform gyrus was found to be sensitive to the relative location of prime and target stimuli, such that priming effects only appeared with centrally located primes. In middle fusiform gyrus, on the other hand, priming effects were insensitive to the relative location of prime and target stimuli. These results therefore suggest that location-specific letter detectors are housed in posterior fusiform, whereas the neurons that perform location-invariant orthographic coding would be situated in more anterior regions. We would therefore speculate that the neural generators of the N/P150 ERP component described in the present work and elsewhere (Chauncey et al., 2008; Holcomb \& Grainger, 2006) are situated in posterior fusiform gyrus. Consistent with this hypothesis are recent ERP and fMRI data from the masked priming paradigm, where pictures of objects were used as stimuli instead of words. As mentioned previously, Eddy et al. (2006), showed a similar early posterior positivity and anterior negativity for repeated compared to unrelated target objects in an ERP study. In a parallel fMRI study (Eddy, Schnyer, Schmid, \& Holcomb, 2007), masked object priming effects were localized to fusiform cortex, suggesting that the neural generators for the early ERP priming effect may be situated in this region. Future tests of this hypothesis could use MEG, a technique that combines the temporal and spatial resolution necessary to localize spatially and temporally focal ERP components, or a combination of EEG and fMRI recordings.
Finally, Grainger, Kiyonaga, and Holcomb (2006) have shown that phonological priming effects (e.g., trane-train vs. trand-train) show up in the later part of the N250 ERP component, peaking about $50 \mathrm{msec}$ later than do orthographic priming effects (see Ferrand \& Grainger, 1993, for behavioral evidence in line with this timing estimate). This would situate the processes involved in prelexical translation of orthography to phonology as lagging only very slightly behind the processes involved in mapping locationspecific letter representations onto a location-invariant orthographic code. One possibility entertained by Grainger, Kiyonaga, and Holcomb (2006) is that location-specific letter representations in the alphabetic array send activation simultaneously to coarse-grained orthographic representations (e.g., open bigrams) on the one hand, and to finegrained graphemic representations on the other. Grapheme representations require accurate information about letter order that is lacking in the coarse-grained code. It is these grapheme representations that would enable rapid activation of a prelexical phonological code upon presentation of a printed word stimulus.

In conclusion, the results of the present study support the distinction between a location-specific and a locationinvariant (word-centered) orthographic code highlighted in Grainger and van Heuven's (2003) account of orthographic processing, and suggests that this shift from a retinotopic to a nonretinotopic map occurs between 150 and $250 \mathrm{msec}$ poststimulus onset during visual word recognition.

\section{AUTHOR NOTE}

We thank Stéphanie Massol for her help in data collection. This research was supported by Grants ANR-06-BLAN-0337 (Agence Nationale de la Recherche, 2006), HD25889, and HD043251. Correspondence concerning this article should be addressed to S. Dufau, LPC-CNRS/ Université de Provence, 3 place Victor Hugo, 13331 Marseille, France (e-mail: stephane.dufau@univ-provence.fr).

\section{REFERENCES}

American Clinical Neurophysiology Society (2006). Guideline 5: Guidelines for standard electrode position nomenclature. Journal of Clinical Neurophysiology, 23, 107-110.

Caramazza, A., \& Hillis, A. E. (1990). Levels of representation, coordinate frames, and unilateral neglect. Cognitive Neuropsychology, 7, 391-445.

Chauncey, K., Holcomb, P. J., \& Grainger, J. (2008). Effects of stimulus font and size on masked repetition priming: An ERP investigation. Language \& Cognitive Processes, 23, 183-200.

Cohen, L., Dehaene, S., Naccache, L., Lehéricy, S., DehaeneLambertz, G., HénAfF, M. A., \& Michel, F. (2000). The visual word form area: Spatial and temporal characterization of an initial stage of reading in normal subjects and posterior split-brain patients. Brain, 123, 291-307.

Dehaene, S., Cohen, L., Sigman, M., \& Vinckier, F. (2005). The neural code for written words: A proposal. Trends in Cognitive Sciences, 9, 335-341.

Dehaene, S., Jobert, A., Naccache, L., Ciuciu, P., Poline, J., Le Bihan, D., \& Cohen, L. (2004). Letter binding and invariant recognition of masked words: Behavioral and neuroimaging evidence. Psychological Science, 15, 307-313.

Delorme, A., \& MAKEIG, S. (2004). EEGLAB: An open source toolbox for analysis of single-trial EEG dynamics including independent component analysis. Journal of Neuroscience Methods, 134, 9-21.

EDDY, M. D., Schmid, A., \& Holcomb, P. J. (2006). Masked repetition priming and event-related brain potentials: A new approach for 
tracking the time-course of object perception. Psychophysiology, 43, 564-568.

Eddy, M. D., Schnyer, D., Schmid, A., \& Holcomb, P. J. (2007). Spatial dynamics of masked picture repetition effects. NeuroImage, 34, 1723-1732.

Ferrand, L., \& Grainger, J. (1993). The time course of orthographic and phonological code activation in the early phases of visual word recognition. Bulletin of the Psychonomic Society, 31, 119-122.

Grainger, J., Granier, J. P., Farioli, F., Van Assche, E., \& van HeuVEN, W. (2006). Letter position information and printed word perception: The relative-position priming constraint. Journal of Experimental Psychology: Human Perception \& Performance, 32, 865-884.

Grainger, J., \& Holcomb, P. J. (in press). Neural constraints on a functional architecture for word recognition. In P. Cornelissen, P. Hansen, M. Kringelbach, \& K. Pugh (Eds.), The neural basis of reading. Oxford: Oxford University Press.

Grainger, J., Kiyonaga, K., \& Holcomb, P. J. (2006). The time-course of orthographic and phonological code activation. Psychological Science, 17, 1021-1026.

Grainger, J., \& van Heuven, W. (2003). Modeling letter position coding in printed word perception. In P. Bonin (Ed.), The mental lexicon (pp. 1-23). Hauppause, NY: Nova Science.

Holcomb, P. J., \& Grainger, J. (2006). On the time-course of visual word recognition: An event related potential investigation using masked repetition priming. Journal of Cognitive Neuroscience, 18, 1631-1643.

Holcomb, P. J., Reder, L., Misra, M., \& Grainger, J. (2005). The effects of prime visibility on ERP measures of masked priming. Cognitive Brain Research, 24, 155-172.

Jennings, J. R., \& Wood, C. C. (1976). The adjustment procedure for repeated measures analyses of variance. Psychophysiology, 13, 277-278.

Kiyonaga, K., Grainger, J., Midgley, K. J., \& Holcomb, P. J. (2007). Masked cross-modal repetition priming: An event-related potential investigation. Language \& Cognitive Processes, 22, 337-376.

Marzouki, Y., \& Grainger, J. (2008). Effects of prime and target eccentricity on masked repetition priming. Psychonomic Bulletin \& Review, 15, 141-148.

McClelland, J. L., \& Rumelhart, D. E. (1981). An interactive activation model of context effects in letter perception: Part 1. An account of basic findings. Psychological Review, 88, 375-407.

New, B., Pallier, C., Brysbaert, M., \& Ferrand, L. (2004). Lexique 2: A new French lexical database. Behavior Research Methods, Instruments, \& Computers, 36, 516-524.

Petit, J. P., Midgley, K. J., Holcomb, P. J., \& Grainger, J. (2006). On the time course of letter perception: A masked priming ERP investigation. Psychonomic Bulletin \& Review, 13, 674-681.

Schutter, D. J. L. G., Leitner, C., Kenemans, J. L., \& van Honk, J. (2006). Electrophysiological correlates of cortico-subcortical interaction: A cross-frequency spectral EEG analysis. Clinical Neurophysiology, 117, 381-387.

Tydgat, I., \& Grainger, J. (2007). Serial position effects in the identification of letters, digits, and symbols. Manuscript submitted for publication.

Whitney, C. (2001). How the brain encodes the order of letters in a printed word: The SERIOL model and selective literature review. Psychonomic Bulletin \& Review, 8, 221-243.

(Manuscript received July 4, 2007; revision accepted for publication November 18, 2007.) 